\title{
Analysis of gene order data supports vertical inheritance of the leukotoxin operon and genome rearrangements in the 5' flanking region in genus Mannheimia
}

\author{
Jesper Larsen*1, Peter Kuhnert ${ }^{2}$, Joachim Frey ${ }^{2}$, Henrik Christensen ${ }^{1}$, \\ Magne Bisgaard ${ }^{1}$ and John E Olsen ${ }^{1}$
}

Address: ${ }^{1}$ Department of Veterinary Pathobiology, Faculty of Life Sciences, University of Copenhagen, Stigbøjlen 4, DK-1870 Frederiksberg C, Denmark and 2Institute of Veterinary Bacteriology, University of Berne, Länggass-Strasse 122, CH-3012 Berne, Switzerland

Email: Jesper Larsen* - jesl@life.ku.dk; Peter Kuhnert - peter.kuhnert@vbi.unibe.ch; Joachim Frey - joachim.frey@vbi.unibe.ch; Henrik Christensen - hech@life.ku.dk; Magne Bisgaard - mbi@life.ku.dk; John E Olsen - jeo@life.ku.dk

* Corresponding author

Published: 3 October 2007

BMC Evolutionary Biology 2007, 7:184 doi:10.1/86/147/-2/48-7-184

This article is available from: http://www.biomedcentral.com/I47I-2I 48/7//84

(c) 2007 Larsen et al.; licensee BioMed Central Ltd.

This is an Open Access article distributed under the terms of the Creative Commons Attribution License (http://creativecommons.org/licenses/by/2.0), which permits unrestricted use, distribution, and reproduction in any medium, provided the original work is properly cited.

\begin{abstract}
Background: The Mannheimia subclades belong to the same bacterial genus, but have taken divergent paths toward their distinct lifestyles. For example, M. haemolytica + M. glucosida are potential pathogens of the respiratory tract in the mammalian suborder Ruminantia, whereas $M$. ruminalis, the supposed sister group, lives as a commensal in the ovine rumen. We have tested the hypothesis that vertical inheritance of the leukotoxin (lktCABD) operon has occurred from the last common ancestor of genus Mannheimia to any ancestor of the diverging subclades by exploring gene order data.
\end{abstract}

Results: We examined the gene order in the 5 ' flanking region of the leukotoxin operon and found that the $5^{\prime}$ flanking gene strings, $h s / V U$-lapB-art-lktC and xylAB-lktC, are peculiar to M. haemolytica + $M$. glucosida and M. granulomatis, respectively, whereas the gene string hs/VU-lapB-lktC is present in $M$. ruminalis, the supposed sister group of $M$. haemolytica $+M$. glucosida, and in the most ancient subclade $M$. varigena. In M. granulomatis, we found remnants of the gene string hsIVU-lapB-lktC in the $x y l B-l k t C$ intergenic region.

Conclusion: These observations indicate that the gene string hsIVU-lapB-lktC is more ancient than the $h s \mid V U$-lapB-art-lktC and $x y \mid A B-l k t C$ gene strings. The presence of (remnants of) the ancient gene string $h s / V U$-lapB-lktC among any subclades within genus Mannheimia supports that it has been vertically inherited from the last common ancestor of genus Mannheimia to any ancestor of the diverging subclades, thus reaffirming the hypothesis of vertical inheritance of the leukotoxin operon. The presence of individual 5 ' flanking regions in $M$. haemolytica $+M$. glucosida and $M$. granulomatis reflects later genome rearrangements within each subclade. The evolution of the novel 5 ' flanking region in $M$. haemolytica $+M$. glucosida resulted in transcriptional coupling between the divergently arranged art/ and lkt promoters. We propose that the chimeric promoter have led to high level expression of the leukotoxin operon which could explain the increased potential of certain M. haemolytica + M. glucosida strains to cause a particular type of infection. 


\section{Background}

Genomes are evolutionary unstable structures that are subject to frequent dynamic rearrangements [1-4]. Huynen and Bork [5] showed that overall gene order and evolutionary distance are inversely correlated until a saturation level is reached. In a more recent analysis [4] 25 genomes were screened looking for conservation of gene order. This analysis suggested that the saturation level is dictated by functional constraints on operon structure and that only $5-25 \%$ of the genes in bacterial and archaeal genomes belong to gene strings that are conserved in at least two genomes. However, some phylogenetic lineages show remarkable conservation and others extensive genome rearrangements [6,7]. There are four types of changes that are thought to affect the order of the genes on the bacterial genome: (i) inversions and translocations are frequently detected when the genomes of even closely related species are compared [8]; (ii) genes may be lost in a single event [9] or as a consequence of multistep deletion of pseudogenes $[10,11]$; (iii) incorporation of nonhomologous genes or operons throughout the genome by illegitimate recombination in a process known as horizontal gene transfer (HGT) is thought to be a major force in the evolution of gene content of bacterial species $[12,13]$; (iv) partial duplications of the genome may produce redundant genome segments [14-17].

The genus Mannheimia includes strains previously classified as trehalose-negative [Pasteurella] haemolytica and is one of the most well-defined and robust clusters within the gamma-proteobacterial family of Pasteurellaceae Pohl 1981 [18]. The Mannheimia subclades have taken divergent paths toward their distinct lifestyles. For example, $M$. haemolytica $+M$. glucosida form one of the most recently diverged subclades within genus Mannheimia based on $16 S$ rRNA sequences and both species are potential pathogens in the mammalian suborder Ruminantia, whereas M. ruminalis, the supposed sister group, lives as a commensal in the ovine rumen [18]. Previous works have revealed that the leukotoxin (LktA) protein of $M$. haemolytica plays an important role in the evasion and exploitation of the adaptive immune system during pulmonary infection via interactions with host cells [19-21]. The leukotoxin $(l k t)$ operon codes for four proteins: an internal acyltransferase, encoded by $l k t C$ [22]; the structural toxin, encoded by lktA, which belongs to the Escherichia coli HlyA-like subfamily of cytotoxic RTX (repeats in toxin) proteins [22]; an inner membrane protein with a cytoplasmic ATP binding cassette (ABC) domain, encoded by lktB, which pumps out LktA protein via interaction with the $\mathrm{C}$ terminus of LktA [23]; and a membrane fusion protein, encoded by $l k t D$, which forms a bridge between the inner and outer membranes [23]. The genes for these four proteins are physically adjacent on the chromosome and are transcribed as $l k t C A$ or $l k t C A B D$ messages [24,25].
In a recent work, Larsen et al. [26] performed phylogenetic and compositional analyses in order to explore the origin and fate of the lkt operon in M. haemolytica + M. glucosida. The scenario derived from this work suggested that it has been vertically inherited from the last common ancestor of genus Mannheimia to any ancestor of the diverging subclades, although several strains belonging to M. ruminalis have lost the operon. However, reconstruction of the rtxA tree provided strong support for a sister group relationship between $M$. haemolytica $+M$. glucosida and $M$. ruminalis on one hand and the more distantly related taxon [Pasteurella] trehalosi on the other. This incongruence between gene trees and organismal phylogenies could arise from HGT of the lkt operon from $[P$.] trehalosi to a common ancestor of these Mannheimia subclades.

In this article, we have examined the compatibility of the gene order in the 5' flanking region of the lkt operon (called hereafter the 5' flanking gene order) in the genomes of representatives from the four major subclades within genus Mannheimia with the Larsen and coworkers' [26] hypothesis of vertical inheritance. Under the assumption that closely related species accumulate fewer rearrangements than the distant ones, vertical inheritance should result in $l k t$ operons belonging to gene strings that are conserved in all genomes. On the other hand, HGT would result in disruption of the 5 ' flanking gene order (breakpoints) between the same genomes.

\section{Results}

\section{Sequencing of 5' flanking regions}

The 5 ' flanking gene string in M. haemolytica serotype 1 str. PHL101 [GenBank:M59210] contains four genes considered to code for functionally and physically interacting proteins in an L-arginine ABC transporter [27]. In order to examine the compatibility of the 5 ' flanking gene order with the Larsen and coworkers' [26] hypothesis of vertical inheritance, we here analyzed homologous genome segments in representatives from the four major subclades within genus Mannheimia. We retrieved data (contig74) from the annotated genome sequence of $M$. haemolytica serotype 1 str. PHL213 [GenBank:NZ AASA01000031]. For the remaining Mannheimia strains, genome segments were first cloned into the Zap Express vector and packaged into the Gigapack III Gold Packaging Extract. The resulting phagemid libraries were then screened for segments of $5^{\prime}$ flanking genes by the plaque hybridization method using a 542 bp fragment of the lktCA genes as a probe.

The sizes of the sequenced genome segments varied from 1,383 to 9,126 bp (Table 1). Our initial search for putative orthologs suggested that the 5' flanking gene strings, hslVU-lapB-artI/artJ-lktC and $x y l A B-l k t C$, are peculiar to $M$. haemolytica + M. glucosida and M. granulomatis, respec- 
Table I: Strains used in this study

\begin{tabular}{|c|c|c|c|c|c|c|}
\hline Subclade & Taxon $^{\mathrm{a}}$ & Strain ID & Host & Country & $\begin{array}{r}\text { Size of genome } \\
\text { segment (bp) }\end{array}$ & GenBank \\
\hline & M. haemolytica (Mh) & & & & & \\
\hline I & Biogroup I & PHL2I3 & Ruminantia & & & [GenBank:NZ AASA0100003I] \\
\hline I & $\begin{array}{l}\text { Biogroup I } \\
\text { M. glucosida (Mgl) }\end{array}$ & CCUG $12392^{\top}$ & Ruminantia & UK & 1,383 & [GenBank:AY425276] \\
\hline I & $\begin{array}{l}\text { Biogroup 3B } \\
\text { M. ruminalis (Mr) }\end{array}$ & $\mathrm{P} 925^{\mathrm{T}}$ & Ruminantia & Scotland & 3,373 & [GenBank:AY425277] \\
\hline II & $\begin{array}{l}\text { Bt } 18 \text { biovar } 2 \\
\text { M. granulomatis (Mgr) }\end{array}$ & HPAII3 & Ruminantia & UK & 9,126 & [GenBank:AY425280] \\
\hline III & $\begin{array}{l}{[P .] \text { granulomatis }} \\
\text { M. varigena }(\mathrm{Mv})\end{array}$ & $\mathrm{PII35/26^{ \top }}$ & Ruminantia & Brazil & 5,233 & [GenBank:AY425278] \\
\hline IV & Biogroup 6 & $177^{\top}$ & Ruminantia & Germany & 4,765 & [GenBank:AY425279] \\
\hline
\end{tabular}

$\mathrm{a} \mathrm{Bt}=$ Bisgaard taxon.

tively, whereas the gene string hslVU-lapB-lktC is present in $M$. ruminalis, the supposed sister group of $M$. haemolyt$i c a+M$. glucosida, and in the most ancient subclade M. varigena (Figure 1). It should be noted that for one open reading frame (ORF) we retrieved two closely related proteins, ArtI and ArtJ, in a subset of the genomes and that the corresponding artI/artJ gene is located on the opposite strand immediately upstream of the $l k t$ operon.

\section{Description of genes}

Prior to this work, Highlander et al. [27] analyzed the 5' flanking genes in M. haemolytica serotype 1 str. PHL101 and noted that the gene cluster lapCABT, corresponding to the gene string $h s l V$-hslU-lapB-artI/artJ, codes for functionally and physically interacting proteins in an L-arginine $\mathrm{ABC}$ transporter. A close look at the Lap proteins showed that updated annotations were already available in SWISS-PROT (Table 2). The SWISS-PROT entry for the LapT protein [SWISS-PROT:P49618] showed that it is annotated as "arginine-binding periplasmic protein". PSIBLAST searches of the COG database revealed that LapT belongs to COG0834 (annotated as "ABC-type amino acid transport/signal transduction systems, periplasmic component/domain"), thus supporting the updated annotation in SWISS-PROT (Table 2). Our initial search for putative orthologs retrieved two closely related proteins, ArtI and ArtJ, in a subset of the genomes as explained above. These proteins belong to the polar amino acid transporter (PAAT) family of the periplasmic binding protein clan [28]. Members of this family bind substrates in the vicinity of the inner membrane with high affinity followed by delivery of the liganded form to a membrane bound complex for translocation. These complexes belong to the family of $\mathrm{ABC}$ transporters and are composed of two integral membrane domains and two hydrophilic domains that each contains a conserved nucleotide binding domain (NBD). The different domains are composed of separate protein subunits in prokaryotes, whereas they are generally fused into a single polypeptide in eukaryotes. In E. coli, the membrane bound complex of the L-arginine $\mathrm{ABC}$ transporter ArtQMP $_{2}$ consists of two integral membrane protein subunits, ArtQ and ArtM, and two copies of the nucleotide binding protein subunit ArtP [29]. Previous works revealed that ArtJ from E. coli and LapT from M. haemolytica serotype 1 str. PHL101 bind L-arginine with a higher affinity compared to other amino acids based on equilibrium titration experiments [29,30]. On the contrary, the binding affinity for L-arginine by ArtI from E. coli was one order of magnitude lower compared to ArtJ and was almost identical to the binding affinities for the other amino acids by ArtI and ArtJ [29]. These data suggest that LapT and ArtJ, but not ArtI, are orthologs of each other.

The LapB protein [SWISS-PROT:P32181] is annotated as "membrane protein LapB" in SWISS-PROT. PSI-BLAST searches of the COG database showed that LapB belongs to COG2990 (annotated as "uncharacterized protein conserved in bacteria") (Table 2). These data support that the lapB gene could code for an integral membrane protein subunit (ArtQ or ArtM) of an L-arginine ABC transporter as predicted by Highlander et al. [27], but other alternatives has to be considered.

The LapC protein [SWISS-PROT:P49617] has been annotated as "ATP-dependent protease HslV", whereas the LapA protein [SWISS-PROT:P32180] has been annotated as "ATP-dependent Hsl protease ATP-binding subunit HslU" in SWISS-PROT. PSI-BLAST searches of the COG database revealed that LapC and LapA belong to COG5405 (annotated as "ATP-dependent protease HsIVU (ClpYQ), peptidase subunit") and COG1220 (annotated as "ATP-dependent protease HsIVU (ClpYQ), ATPase subunit"), respectively, thus reaffirming the updated annotations in SWISS-PROT (Table 2). The bacterial ATPdependent protease HsIVU is a homolog of the eukaryotic 


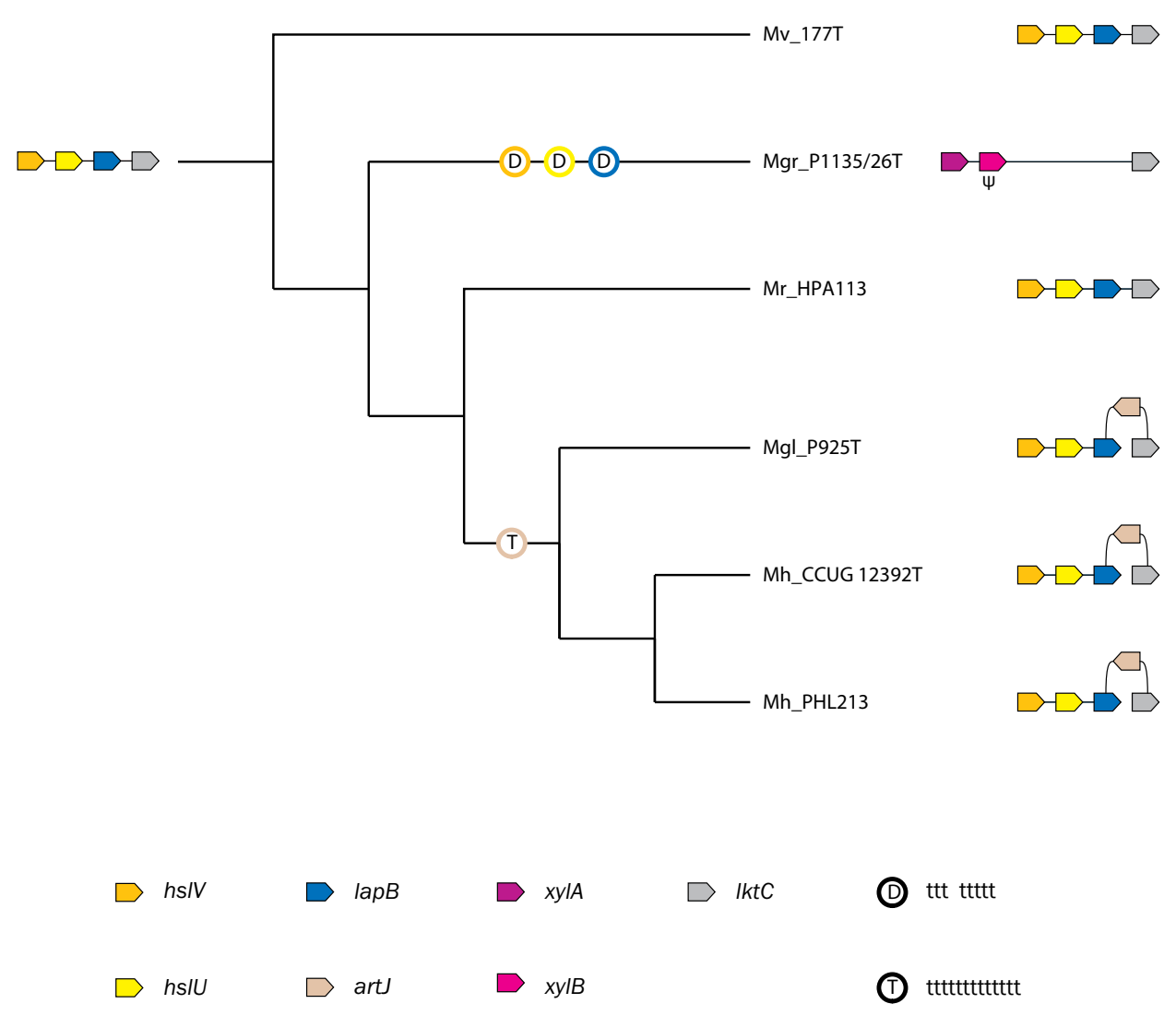

\section{Figure I}

Evolution of the 5' flanking gene order in the Mannheimia genomes analyzed in this study. The organismal cladogram that describes bifurcation order was adapted from Larsen et al. [26]. Colored arrows indicate orthology as in Figure 2. Gene names are those reported in protein databases or have been assigned by us on the basis of orthology relationships. Pseudogenes are indicated by a " $\Psi$ ". The two types of genome rearrangements are indicated by circles. Nomenclature: sequence names contain abbreviations of the taxonomic group (genus and species) followed by the corresponding strain ID as listed in Table I.

26S proteasome [31]. The proteolytic subunit HslV (ClpQ) is a member of the MEROPS peptidase subfamily T01B [32]. Structural information revealed that the HslV protein from E. coli and the $\beta$-subunits of the proteasome catalytic core (20S particle) share $20 \%$ similarity over $\sim 200$ amino acid residues and a conserved fold [33]. HslU
(ClpY) belongs to the Clp/Hsp100 proteins of the AAA+ superfamily which comprises ClpA, ClpX, and HslU on one hand and ClpB on the other [34]. ClpA, ClpX, and HslU interact with cellular peptidases to form ATPdependent proteases. The role of these Clp proteins is the unfolding of substrates by energy-dependent threading

Table 2: Homology-based functional characterization of the $5^{\prime}$ flanking genes

\begin{tabular}{|c|c|c|c|c|}
\hline Ortholog & Size (aa) & SWISS-PROT & COG & COG annotation \\
\hline hsIV & 173 & [SWISS-PROT:P49617] & 5405 & ATP-dependent protease HsIVU (ClpYQ), peptidase subunit \\
\hline hslU & 440 & [SWISS-PROT:P32180] & 1220 & ATP-dependent protease HsIVU (ClpYQ), ATPase subunit \\
\hline$l a p B$ & 225 & [SWISS-PROT:P32181] & 2990 & Uncharacterized protein conserved in bacteria \\
\hline artl/art] & 237 & [SWISS-PROT:P49618] & 0834 & $\begin{array}{l}\text { ABC-type amino acid transport/signal transduction systems, periplasmic } \\
\text { component/domain }\end{array}$ \\
\hline$x y \mid A$ & 439 & & 2115 & Xylose isomerase \\
\hline$x y / B$ & 484 & & 1070 & Sugar (pentulose and hexulose) kinases \\
\hline
\end{tabular}


prior to the delivery to the proteolytic subunit. On the contrary, ClpB from E. coli and Thermus thermophilus and the homolog Hsp104 from Saccharomyces cerevisiae do not appear to bind to cellular proteases. Instead they interact with the DnaK/Hsp70 chaperone system to assist in the disaggregation and reactivation of strongly aggregated proteins [35-39]. Clp proteins can be further classified according to the number of NBDs which contain the ATP binding Walker A and B motifs. Class 1 proteins ClpA and ClpB contain two NBDs, whereas class 2 proteins HslU and ClpX contain only one NBD $[40,41]$. Sequence and structural information has shed light on the domain architecture of the class 2 protein HslU from E. coli $[34,42]$. This protein is folded into three distinct domains with the $\mathrm{N}$ domain comprising residues 2-109 and 244-332 and the $\mathrm{C}$ domain comprising residues 333-443. The intermediate domain (I domain) that is not found in other $\mathrm{AAA}+$ proteins emerges from the $\mathrm{N}$ domain at residue 110 and returns to it at residue 243 . Multiple alignment of Clp proteins from E. coli showed that the spacing between the Walker A and B motifs in ClpX and ClpA is 55 residues, whereas it is 186 residues in HslU due to their separation by the I domain [43]. PSIBLAST searches of the PDB database and the corresponding multiple alignments showed that the domain architecture of LapA and HslU is conserved, including the spacing between the Walker A and B motifs (184 and 186 residues, respectively) (data not shown). Highlander et al. [27] used homology methods to compare the LapA protein with the Clp/Hsp100 protein ClpA (class 1 protein containing two NBDs), but not HslU (class 2 protein containing only one NBD), and nucleotide binding protein subunits of the ABC transporter family (each containing one NBD). These authors classified LapA as the nucleotide binding protein subunit of an L-arginine $\mathrm{ABC}$ transporter (ArtP) based on the spacing between the Walker A and $\mathrm{B}$ motifs and the number of NBDs. However, the spacing between these motifs in the closely related nucleotide binding protein subunits ArtP and HisP is only 118 and 126 residues, respectively $[29,44]$, whereas it is 184 residues in LapA and 186 residues in HslU as explained above. These data suggest that LapA and HslU are putative orthologs of each other, thus rejecting the Highlander and coworkers' [27] hypothesis that the lapA gene codes for a nucleotide binding protein subunit (ArtP) of an Larginine $\mathrm{ABC}$ transporter.

PSI-BLAST searches of the COG database showed that the XylA and XylB proteins belong to COG2115 (annotated as "xylose isomerase") and COG1070 (annotated as "sugar (pentulose and hexulose) kinases"), respectively (Table 2). Two previous works have revealed that these proteins are involved in the dissimilation of D-xylose through the pentose phosphate pathway in E. coli and Salmonella typhimurium $[45,46]$. The sugar is first isomerized into D-xylulose by xylose isomerase (XylA), which is a member of the xylose isomerase-like TIM barrel family, and then phosphorylated by xylulokinase (XylB), which belongs to the FGGY family of carbohydrate kinases, to produce D-xylulose 5-phosphate.

\section{Description of pseudogenes and remnant DNA}

The $x y l B$ gene in M. granulomatis str. P1135/26 $6^{\mathrm{T}}$ is predicted to code for a short peptide that corresponds to $\sim 57 \%$ of the XylB protein. The premature stop codon is located at codon 276 and is the result of frameshift due to a $1 \mathrm{bp}$ insertion at the first position of codon 270 (data not shown). The designation of $x y l B$ as a pseudogene is compatible with the results of Angen et al. [47], who showed that a single lineage of $M$. granulomatis, including strain P1135/26T, has lost the ability to utilize D-xylose as a carbon source.

Interestingly, the lapB gene in $M$. haemolytica serotype 1 str. PHL101 is predicted to code for a short peptide that corresponds to the first $\sim 75 \%$ of the LapB protein (data not shown). Multiple alignment revealed that the pseudogene originates from a 1 bp deletion at the second position of codon 217 leading to frameshift and a premature stop codon at codon 225 (data not shown).

If we assume that genes have been inactivated continuously since the divergence of M. granulomatis, it is expected that some genes have been degraded to such an extent that they are no longer recognizable as pseudogenes. In order to explore the fate of the genes identified in the other Mannheimia genomes in the previous step, we performed BLAST searches of the $x y l B-l k t C$ intergenic region against 5 ' flanking gene strings in these genomes. Interestingly, we found a weak but significant hit to the hslVU operon in $M$. haemolytica serotype 1 str. PHL213 (E-value = 5E-16) due to a $50 \mathrm{bp}$ inverted sequence (data not shown).

\section{Description of conserved gene strings}

Based on the notion that statistically significant conserved gene strings can be confidently predicted to form operons [4], we have analyzed a set of 17 proteobacterial genomes in order to determine the operon organization of the $5^{\prime}$ flanking genes. The data set included a balanced number of representatives from the gamma-proteobacterial group, including three Pasteurellaceae genomes which are closely related to the Mannheimia genomes, and the beta-proteobacterial sister group (Table 3 ).

The $h s l V$ and hslU genes from E. coli are transcribed from a single promoter containing recognition signals for the heat shock transcription factor $\sigma^{32}$, with consensus sequence STTGAA- $\mathrm{N}_{11-12}$-GNCCCCATWT, suggesting that the genes belong to the $\sigma^{32}$ regulon [42]. STRING searches revealed that the $h s l V$ and hslU genes are present 
Table 3: Proteobacterial genomes analyzed in this study

\begin{tabular}{|c|c|c|c|c|}
\hline Organism & Class & Order & Family & GenBank \\
\hline Bordetella bronchiseptica RB50 & Beta & Burkholderiales & Alcaligenaceae & [GenBank:BX470250] \\
\hline Ralstonia solanacearum GMI000 & Beta & Burkholderiales & Burkholderiaceae & [GenBank:AL646052] \\
\hline Neisseria meningitidis serogroup A strain Z249I & Beta & Neisseriales & Neisseriaceae & [GenBank:ALI57959] \\
\hline Nitrosomonas europaea ATCC 19718 & Beta & Nitrosomonadales & Nitrosomonadaceae & [GenBank:AL954747] \\
\hline Shewanella oneidensis MR-I & Gamma & Alteromonadales & Shewanellaceae & [GenBank:AE0|4299] \\
\hline Buchnera aphidicola str. APS & Gamma & Enterobacteriales & Enterobacteriaceae & [GenBank:BA000003] \\
\hline Escherichia coli OI 57:H7 str. Sakai & Gamma & Enterobacteriales & Enterobacteriaceae & [GenBank:BA000007] \\
\hline Photorhabdus luminescens subsp. laumondii TTOI & Gamma & Enterobacteriales & Enterobacteriaceae & [GenBank:BX47025I] \\
\hline Salmonella enterica serovar Typhi strain Ty2 & Gamma & Enterobacteriales & Enterobacteriaceae & [GenBank:AE0|46|3] \\
\hline Coxiella burnetii RSA 493 & Gamma & Legionellales & Coxiellaceae & [GenBank:AE016828] \\
\hline Haemophilus ducreyi $35000 \mathrm{HP}$ & Gamma & Pasteurellales & Pasteurellaceae & [GenBank:AE0|7|43] \\
\hline Haemophilus influenzae Rd KW20 & Gamma & Pasteurellales & Pasteurellaceae & [GenBank:L42023] \\
\hline Pasteurella multocida PM70 & Gamma & Pasteurellales & Pasteurellaceae & [GenBank:AE004439] \\
\hline Pseudomonas aeruginosa PAOI & Gamma & Pseudomonadales & Pseudomonadaceae & [GenBank:AE00409I] \\
\hline Vibrio vulnificus YJOI6 & Gamma & Vibrionales & Vibrionaceae & $\begin{array}{l}{[\text { GenBank:BA000037] }} \\
{[\text { GenBank:BA000038] }}\end{array}$ \\
\hline $\begin{array}{l}\text { Xanthomonas campestris pv. campestris str. ATCC } \\
33913\end{array}$ & Gamma & Xanthomonadales & Xanthomonadaceae & [GenBank:AE008922] \\
\hline Xylella fastidiosa $9 \mathrm{a} 5 \mathrm{c}$ & Gamma & Xanthomonadales & Xanthomonadaceae & [GenBank:AE003849] \\
\hline
\end{tabular}

in 15 genomes, including those of Pasteurellaceae, where they belong to the conserved gene string hslV-hslU, suggesting that these genes comprise an operon (Figure 2). We have shown that the $h s l V$ and $h s l U$ genes from $M$. haemolytica serotype 1 str. PHL213 are transcribed in the same direction (Figure 1) and that putative -10 and -35 motifs for $\sigma^{32}$ are present upstream of $h s l V$ (data not shown). These results are compatible with the predictions of Chuang et al. [42] and provide clear and robust support for functional and physical interactions between the hslV and $h s l U$ genes.

The lapB gene is present in eight of the genomes, including those of Pasteurellaceae, but does not belong to any gene string spanning two or more genes, suggesting that the gene comprises its own operon (Figure 2). The lapB gene was represented by a pseudogene in $M$. haemolytica serotype 1 str. PHL101 as explained above. However, Caskey et al. [30] have shown that this strain is able to transport L-arginine with a high efficiency, something that seems unlikely if LapB constitutes an integral membrane protein subunit (ArtM or ArtQ) of an L-arginine ABC transporter as predicted by Highlander et al. [27]. These results support that the lapB operon is functionally unrelated to the other lap genes.

Two previous works have revealed that the genes encoding the different subunits of the L-arginine $\mathrm{ABC}$ transporter $\operatorname{ArtQMP}_{2}$ and the periplasmic binding proteins, ArtI and ArtJ, belong to the gene string artP-artI-artQ-artM-artJ in $E$. coli $[29,48]$. Although the genes code for functionally and physically interacting proteins, the results of Wissenbach et al. [48] suggest that they are organized into two separate operons: artPIQM and artJ. The results obtained from STRING searches highlighted two aspects (Figure 2). On one hand, the artI gene is present in six genomes, including those of Pasteurellaceae, where it belongs to the conserved gene string artP-artI-artQ-artM. On the other hand, the artJ gene is present in only the enterobacterial genomes of E. coli O157:O7 str. Sakai and S. Typhi strain Ty2 where it is connected to the conserved gene string artP-artI-artQ-artM. These results are compatible with our initial search for putative orthologs which retrieved two closely related proteins, ArtI and ArtJ, in a subset of the genomes, suggesting that the artJ gene is absent from most genomes. For each art gene we searched the genome of $M$. haemolytica serotype 1 str. PHL213 in order to identify putative orthologs. We found a main cluster of typical genes, artP-artI/artJ-artQ-artM, on contig22 and a single gene, lapT, on contig74 (data not shown). Highlander et al. [27] have shown that lapT is transcribed from a single promoter, suggesting that the gene comprises its own operon, while we have shown that the identified genes on contig22 are transcribed in the same direction (data not shown). These results are compatible with the predictions of Wissenbach and coworkers $[29,48]$ and support that the artPIQM and lapT genes are organized into two separate operons, thus reaffirming our hypothesis that lapT and artJ, but not artI, are orthologs of each other.

The $x y l A$ and $x y l B$ genes were predicted to code for proteins that functionally interact in the dissimilation of $\mathrm{D}$ xylose through the pentose phosphate pathway. The $x y l A$ and $x y l B$ genes from $E$. coli are organized into a single operon [49]. STRING searches revealed that the xylA and $x y l B$ genes are present in four genomes, including only 


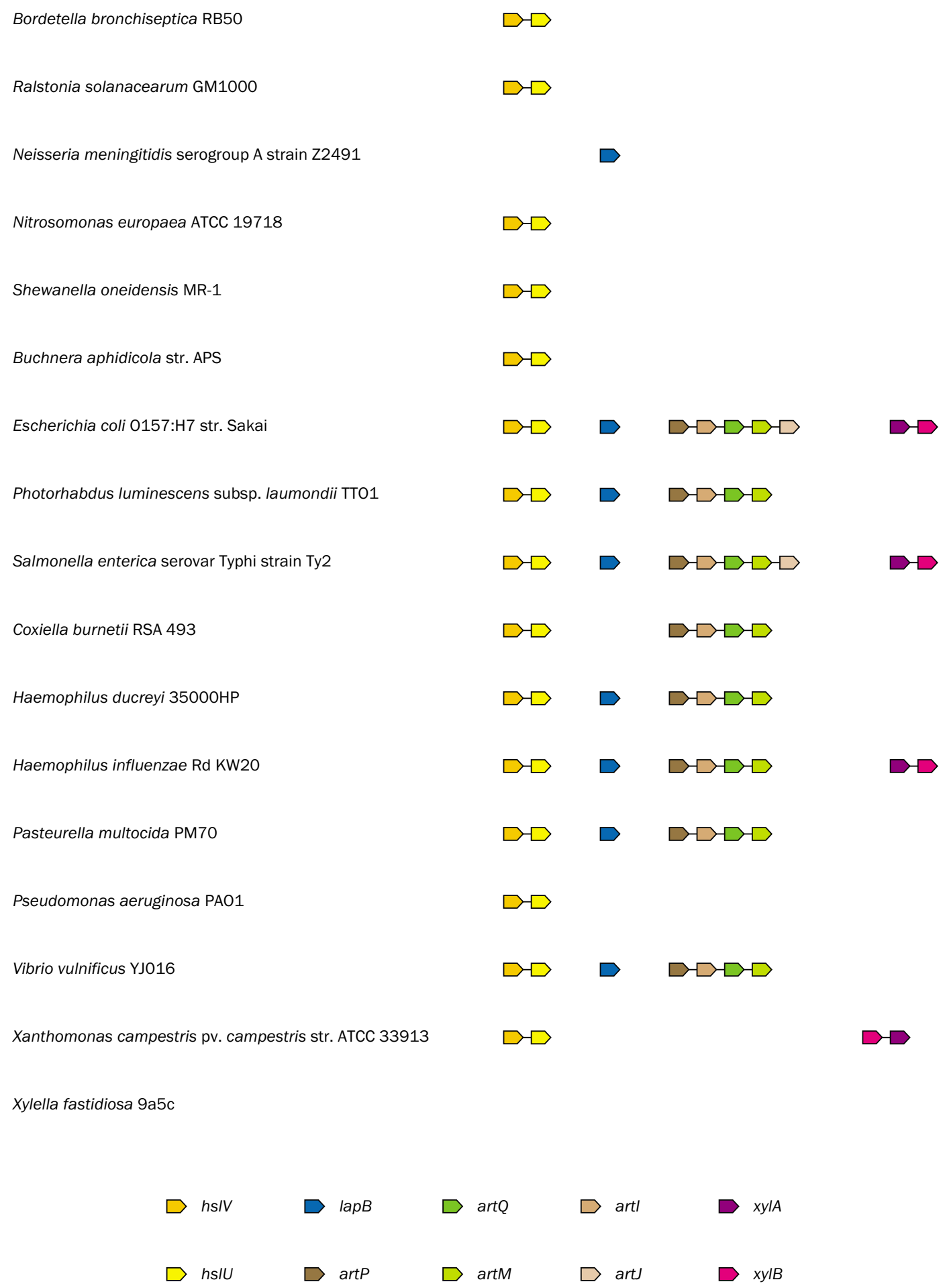

\section{Figure 2}

Conserved gene strings in 17 prokaryotic genomes analyzed in this study. For each protein searches were performed at the STRING server [57] with a high confidence score $>0.7$. Colored arrows indicate orthology. Gene names are those reported in protein databases. 
one Pasteurellaceae genome, where they belong to the conserved gene string $x y l A-x y l B$ (Figure 2). In accordance with these results, we have shown that the $x y l A$ and $x y l B$ genes are transcribed in the same direction (Figure 1), supporting that the respective genes comprise an operon.

\section{Discussion}

Table 1 presents the Mannheimia genomes analyzed in this study, sorted by their overall similarity to M. haemolytica serotype 1 str. PHL213. The dataset includes the type strains of each species, except M.ruminalis where the type strain HPA92 ${ }^{\mathrm{T}}$ has lost the lkt operon [26], and represents the diversity within genus Mannheimia based on phenotypic characters (biogroups), 16S rRNA phylogeny, and geographic origin. Most Mannheimia species are inhabitants of the respiratory tract, but the genomes analyzed here encompass a wide variety of lifestyles, including potential pathogens of the ruminant respiratory tract $(M$. haemolytica + M. glucosida and M. varigena), one commensal of the ovine rumen (M. ruminalis), and one potential pathogen of the ruminant integument (M. granulomatis).

Prior to this work, Larsen et al. [26] used a strategy that combines phylogenetic and compositional methods to explore the evolution of the lkt operon in genus Mannheimia. This analysis suggested that the operon has been vertically inherited from the last common ancestor of genus Mannheimia to any ancestor of the diverging subclades, although several strains belonging to $M$. ruminalis have lost the operon. However, reconstruction of the $r t x A$ tree provided strong support for a sister group relationship between $M$. haemolytica + M. glucosida and M. ruminalis on one hand and the more distantly related taxon [Pasteurella] trehalosi on the other. This incongruence between gene trees and organismal phylogenies could arise from HGT of the $l k t$ operon from [P.] trehalosi to a common ancestor of these Mannheimia subclades.

In this article, we have examined the compatibility of the 5 ' flanking gene order with the Larsen and coworkers' [26] hypothesis of vertical inheritance. Given the collection of strains, information on the operon organization of the $5^{\prime}$ flanking genes, and an organismal phylogeny that describes bifurcation order, the 5' flanking gene order of their ancestors can be inferred. The results of Highlander et al. [27] suggested that the gene cluster lapCABT codes for functionally and physically interacting proteins in an L-arginine ABC transporter from $M$. haemolytica serotype 1 str. PHL101. However, our results indicate that these genes comprise three functionally unrelated operons: hslVU (lapCA), lapB, and artJ (lapT). The most probable evolutionary scenario to explain the phylogenetic origin and distribution pattern of individual 5' flanking regions is shown in Figure 1. The 5' flanking gene strings, hslVUlapB-artJ-lktC and $x y l A B-l k t C$, are peculiar to M. haemolytica
+ M. glucosida and M. granulomatis, respectively, whereas the gene string $h s l V U-l a p B-l k t C$ is present in M. ruminalis, the supposed sister group of M. haemolytica + M. glucosida, and in the most ancient subclade M. varigena. In M. granulomatis, we found remnants of the gene string hslVU-lapB$l k t C$ in the $x y l B$-lktC intergenic region as explained below. These observations indicate that the gene string hslVU$l a p B-l k t C$ is more ancient than the hslVU-lapB-artJ-lktC and $x y l A B-l k t C$ gene strings. The disruption of the 5 ' flanking gene order in the genomes of M. haemolytica $+M$. glucosida can be explained by two different plausible evolutionary scenarios. On one hand, the absence of the art $J$ operon from the closely related Pasteurellaceae genomes and the presence of the operon in the enterobacterial genomes of E. coli O157:O7 str. Sakai and S. Typhi strain Ty2 suggest that it has been acquired by HGT. On the other hand, the insertion of the artJ operon could also be due to translocation of a vertically inherited art operon and parallel loss of the operon in the closely related Pasteurellaceae genomes. In a recent work, Larsen et al. [26] used the average relative 3:1 dinucleotide abundance to identify horizontally transferred genes among a dataset of 56 annotated genes from $M$. haemolytica and noted that the lapT (artJ) gene was non-deviant from the average genome signature, thus opposing the hypothesis of HGT. It should be noted that false negatives (missed transferred genes) arise when genes have ameliorated due to the mutational processes affecting the recipient genome or the genes are closely related to the recipient genome in terms of context bias [50]. However, the young age of such a transfer (i.e. during early evolution of $M$. haemolytica $+M$. glucosida) and the absence of the artJ operon from the closely related Pasteurellaceae genomes make these scenarios unlikely. Therefore, the disruption of the 5' flanking gene order in the genomes of $M$. haemolytica $+M$. glucosida is compatible only with a history of translocation of the artJ operon during early evolution of this subclade (Figure 1). One of the unexpected findings of the first comparisons of the $5^{\prime}$ flanking regions was the presence of a unique $x y l A B$ operon immediately upstream of the lkt operon in $M$. granulomatis which diverged after $M$. varigena but prior to $M$. ruminalis and $M$. haemolytica $+M$. glucosida. We have found a weak but significant hit to the hslVU operon in the $x y l B-l k t C$ intergenic region, reflecting the presence of highly fragmented remnants of the ancient gene string $h s l V U$-lapB-lktC. The scenario derived from this analysis is compatible with a history of inactivation and multistep deletion of the $h s l V, h s l U$, and $l a p B$ genes after divergence from the remaining Mannheimia subclades (Figure 1).

The results of this analysis are compatible with the Larsen and coworkers' [26] hypothesis of vertical inheritance as reflected in Figure 1. Following this hypothesis, under the assumption that closely related species accumulate fewer rearrangements than the distant ones, it is expected that 
the lkt operons belong to gene strings that are conserved in all genomes, whereas HGT would result in disruption of the 5' flanking gene order (breakpoints) between the same genomes. Indeed, we found (remnants of) the ancient gene string $h s l V U$-lapB-lktC among any subclades within genus Mannheimia, suggesting that it has been vertically inherited from the last common ancestor of genus Mannheimia to any ancestor of the diverging subclades. The presence of individual 5 ' flanking regions in $M$. haemolytica + M. glucosida and M. granulomatis reflects later genome rearrangements within each subclade. Thus, the incongruence observed in the $r t x A$ tree [26] points towards two most probable explanations. On one hand, it is compatible with a history of HGT from a common ancestor of $M$. haemolytica $+M$. glucosida and $M$. ruminalis to $[P$.] trehalosi. On the other hand, it may reflect a history of homologous recombination between vertically inherited $l k t A$ genes.

While the set of Mannheimia genomes allowed us to study the phylogenetic origin of the lkt operon, we cannot rule out additional divergences within the 5 ' flanking region at or below the subclade level. For example, the analysis of the promoter activity of the $5^{\prime}$ region in M. haemolytica serotype 1 str. PHL101 has shown that transcriptional coupling between the divergently arranged artJ and $l k t$ promoters is important for coordinating high level expression of the lkt operon [51]. Thus, during early evolution of $M$. haemolytica $+M$. glucosida, the acquisition of a preformed transcriptional enhancer has occurred which could explain the increased potential of certain M. haemolytica + M. glucosida strains to cause a particular type of infection. However, in order to determine whether the evolution of novel 5' flanking regions has contributed to pathoadaptive niche differentiation within genus Mannheimia, we need to examine the distribution pattern of individual $5^{\prime}$ flanking regions by including additional strains belonging to each subclade.

\section{Conclusion}

To sum up, two conclusions have been outlined. On one hand, the presence of (remnants of) the ancient gene string $h s l V U$-lapB-lktC among any subclades within genus Mannheimia supports that it has been vertically inherited from the last common ancestor of genus Mannheimia to any ancestor of the diverging subclades, thus reaffirming the Larsen and coworker's [26] hypothesis of vertical inheritance of the lkt operon On the other hand, acquisition of individual $5^{\prime}$ flanking regions has occurred in $M$. haemolytica + M. glucosida and M. granulomatis.

\section{Methods}

Taxa used

For this study we included M. haemolytica serotype 1 str. PHL213, M. haemolytica serotype 2 str. CCUG 12392T, M. glucosida serotype 11 str. P925T, M. ruminalis str. HPA113, M. granulomatis str. P1135/26 , and M. varigena str. $177^{\mathrm{T}}$ to balance the number of representatives from the four major subclades within genus Mannheimia based on phenotypic characters (biogroups), 16S rRNA phylogeny, geographic origin, and lifestyle (see Table 1 for a description of strains used in this study, along with their accession numbers). All strains in our analysis have been represented in previous studies by Angen et al. $[18,47,52,53]$ and Larsen et al. [26].

\section{DNA isolation, amplification, and sequencing}

We retrieved data (contig74) from the annotated genome sequence of M. haemolytica serotype $1 \mathrm{str}$. PHL213. For the remaining Mannheimia strains, we constructed, screened, and sequenced phagemid libraries. We constructed a 542 bp probe in the lktCA region from $M$. haemolytica serotype 1 str. PHL213 by using the forward primer lktc_UP (5'CCCTCCACAAAGAATGGAGCTG-3') in conjunction with the reverse primer lkta_DOWN (5'-TTTTGGTTGCCGTTAAAGTGTT-3'). The reaction conditions were $2.5 \mathrm{U} \mathrm{Taq}$ polymerase, $16 \mathrm{mM}\left(\mathrm{NH}_{4}\right)_{2} \mathrm{SO}_{4}, 67 \mathrm{mM}$ Tris-HCl, $0.01 \%$ Tween-20, $2.5 \mathrm{mM} \mathrm{Mg}_{2} \mathrm{SO}_{4}$, each primer at $0.5 \mathrm{mM}$, and each nucleotide at $0.1 \mathrm{mM}$. The cycling conditions were initial denaturation at $94^{\circ} \mathrm{C}$ followed by 25 cycles of $94^{\circ} \mathrm{C}$ for $30 \mathrm{~s}, 52^{\circ} \mathrm{C}$ for $30 \mathrm{~s}$, and $72^{\circ} \mathrm{C}$ for $30 \mathrm{~s}$, finishing with extension at $72^{\circ} \mathrm{C}$ for $10 \mathrm{~min}$. The PCR product was labeled with digoxigenin-11-dUTP by using the Random Primed DNA Labeling Kit according to the manufacturer's instructions (Roche). To construct phagemid libraries, we partially digested genomic DNA with the restriction enzyme Sau3AI. Aliquots were run on agarose gels and fragments of approximately 3-6 kb were cloned into the BamHI site of the Zap Express vector according to the manufacturer's instructions (Stratagene). The ligation mixture was packaged in vitro into the Gigapack III Gold Packaging Extract and transfected into E. coli XL1-Blue MRF' according to the manufacturer's instructions (Stratagene). The plaques were lifted and crosslinked to a 0.45 $\mu \mathrm{m}$ nitrocellulose membrane (Millipore) according to the manufacturer's instructions (Stratagene). The phagemid libraries were screened for $l k t C A$ clones and the pBK-CMV phagemid vector was in vivo excised from the ZAP Express vector by using the ExAssist helper phage and E. coli XLOLR according to the manufacturer's instructions (Stratagene). The plasmids were then directly sequenced.

\section{Analysis of genes, pseudogenes, and remnant DNA}

ORFs were considered to be genes if they showed a significant database hit, or were longer than 100 amino acids, or were longer than 50 amino acids and conserved between at least two genomes in the data set. Sequences with database matches to a functional gene but spanning more than one ORF were considered to be pseudogenes. Significant sequence similarities between unique genes or 
gene strings in one genome and intergenic regions located at the corresponding sites in other genomes were considered to be remnant DNA.

For each ORF we searched the NCBI Genome database by using PSI-BLAST [54] with default parameters until convergence in order to identify putative orthologs. BLAST searches were performed with a very stringent cut-off value $(\mathrm{E}=1 \mathrm{E}-50)$ to minimize problems associated to BLAST identifications. The annotation of each sequence and the corresponding multiple alignments were revised individually to discard wrongly identified putative orthologs. Functional characterization of genes was assessed by searching the SWISS-PROT database [55], the Clusters of Orthologous Groups of proteins (COG) database [56], and the Protein Data Bank (PDB) by using PSIBLAST [54] with default parameters until convergence.

Conservation of gene order is one of the principal types of genome context information and is considered to be an indication of operon organization of the respective genes and a legitimate predictor of functional and potentially physical interactions between genes [4]. We selected 17 proteobacterial genomes for analysis of gene order conservation (Table 3 ). The data set included a balanced number of representatives from the gamma-proteobacterial group, including three Pasteurellaceae genomes which are closely related to the Mannheimia genomes, and the beta-proteobacterial sister group. For each protein we performed searches at the STRING server [57] with a high confidence score $>0.7$.

\section{Authors' contributions}

JL carried out the molecular genetic studies, participated in the sequence annotations and in the interpretation of the results, and drafted the manuscript. PK and JF participated in the design of the study. HC participated in the analysis of gene order data. $\mathrm{MB}$ was responsible for the primary strain collection and participated in the interpretation of the results. JEO conceived of the study, participated in its design and coordination, and helped to draft the manuscript. All authors read and approved the final manuscript.

\section{Acknowledgements}

We thank Yvonne Schlatter for technical assistance. This work was supported by a grant from the Danish Agricultural and Veterinary Research Council (9702797).

\section{References}

I. Mushegian AR, Koonin EV: Gene order is not conserved in bacterial evolution. Trends Genet 1996, I 2:289-290.

2. Rogozin IB, Makarova KS, Murvai J, Czabarka E, Wolf YI, Tatusov RL, Szekely LA, Koonin EV: Connected gene neighborhoods in prokaryotic genomes. Nucleic Acids Res 2002, 30:22 I2-2223.

3. Watanabe H, Mori H, Itoh T, Gojobori T: Genome plasticity as a paradigm of eubacteria evolution. J Mol Evol 1997, 44 Suppl I:S57-S64.
4. Wolf YI, Rogozin IB, Kondrashov AS, Koonin EV: Genome alignment, evolution of prokaryotic genome organization, and prediction of gene function using genomic context. Genome Res 200I, I I:356-372.

5. Huynen MA, Bork P: Measuring genome evolution. Proc Natl Acad Sci U S A 1998, 95:5849-5856.

6. Casjens S: The diverse and dynamic structure of bacterial genomes. Annu Rev Genet 1998, 32:339-377.

7. Nadeau JH, Sankoff D: Counting on comparative maps. Trends Genet 1998, 14:495-50I.

8. Hughes D: Evaluating genome dynamics: the constraints on rearrangements within bacterial genomes. Genome Biol 2000, I:REVIEWSO006.

9. Moran NA, Mira A: The process of genome shrinkage in the obligate symbiont Buchneraaphidicola. Genome Biol 200I, 2:RESEARCH0054.

10. Andersson JO, Andersson SG: Pseudogenes, junk DNA, and the dynamics of Rickettsia genomes. Mol Biol Evol 200I, I 8:829-839.

II. Silva FJ, Latorre A, Moya A: Genome size reduction through multiple events of gene disintegration in Buchnera APS. Trends Genet 200I, 17:615-618.

12. Boucher Y, Douady CJ, Papke RT, Walsh DA, Boudreau ME, Nesbo CL, Case RJ, Doolittle WF: Lateral gene transfer and the origins of prokaryotic groups. Annu Rev Genet 2003, 37:283-328.

13. Koonin EV, Makarova KS, Aravind L: Horizontal gene transfer in prokaryotes: quantification and classification. Annu Rev Microbiol 2001, 55:709-742.

14. Achaz G, Netter P, Coissac E: Study of intrachromosomal duplications among the eukaryote genomes. Mol Biol Evol 200I, 18:2280-2288.

15. Achaz G, Rocha EP, Netter P, Coissac E: Origin and fate of repeats in bacteria. Nucleic Acids Res 2002, 30:2987-2994.

16. Levinson G, Gutman GA: Slipped-strand mispairing: a major mechanism for DNA sequence evolution. Mol Biol Evol 1987, 4:203-221.

17. Ohno S: Evolution by gene duplication New York, Springer Verlag; 1970.

18. Angen, Mutters R, Caugant DA, Olsen JE, Bisgaard M: Taxonomic relationships of the [Pasteurella] haemolytica complex as evaluated by DNA-DNA hybridizations and I6S rRNA sequencing with proposal of Mannheimia haemolytica gen. nov., comb. nov., Mannheimia granulomatis comb. nov., Mannheimia glucosida sp. nov., Mannheimia ruminalis sp. nov. and Mannheimia varigena sp. nov. Int J Syst Bacteriol 1999, 49 Pt I:67-86.

19. Highlander SK, Fedorova ND, Dusek DM, Panciera R, Alvarez LE, Rinehart C: Inactivation of Pasteurella (Mannheimia) haemolytica leukotoxin causes partial attenuation of virulence in a calf challenge model. Infect Immun 2000, 68:3916-3922.

20. Petras SF, Chidambaram M, Illyes EF, Froshauer S, Weinstock GM, Reese CP: Antigenic and virulence properties of Pasteurella haemolytica leukotoxin mutants. Infect Immun 1995, 63:1033-1039.

21. Tatum FM, Briggs RE, Sreevatsan SS, Zehr ES, Ling HS, Whiteley LO, Ames TR, Maheswaran SK: Construction of an isogenic leukotoxin deletion mutant of Pasteurella haemolytica serotype $I$ : characterization and virulence. Microb Pathog 1998, 24:37-46.

22. Lo RY, Strathdee CA, Shewen PE: Nucleotide sequence of the leukotoxin genes of Pasteurella haemolytica A I. Infect Immun 1987, 55:1987-1996.

23. Highlander SK, Chidambaram M, Engler MJ, Weinstock GM: DNA sequence of the Pasteurella haemolytica leukotoxin gene cluster. DNA 1989, 8: I5-28.

24. Highlander SK, Engler MJ, Weinstock GM: Secretion and expression of the Pasteurella haemolytica leukotoxin. I Bacteriol 1990, 172:2343-2350.

25. Strathdee CA, Lo RY: Regulation of expression of the Pasteurella haemolytica leukotoxin determinant. J Bacteriol 1989 , 171:5955-5962.

26. Larsen J, Pedersen AG, Christensen H, Bisgaard M, Angen, Ahrens P, Olsen JE: Evidence for vertical inheritance and loss of the leukotoxin operon in genus Mannheimia. J Mol Evol 2007, 64:423-437.

27. Highlander SK, Wickersham EA, Garza O, Weinstock GM: Expression of the Pasteurella haemolytica leukotoxin is inhibited by a locus that encodes an ATP-binding cassette homolog. Infect Immun 1993, 61:3942-395I. 
28. Saier $\mathrm{MH}$ Jr.: A functional-phylogenetic classification system for transmembrane solute transporters. Microbiol Mol Biol Rev 2000, 64:354-4II.

29. Wissenbach U, Six S, Bongaerts J, Ternes D, Steinwachs S, Unden G: A third periplasmic transport system for L-arginine in Escherichiacoli: molecular characterization of the artPIQMJ genes, arginine binding and transport. Mol Microbiol 1995, i 7:675-686.

30. Caskey LS, Lamphear JG, Highlander SK: Binding-protein-dependent arginine transport in Pasteurella haemolytica. Microbiology 1996, 142 ( Pt 7): I739-1747.

31. De Mot R, Nagy I, Walz J, Baumeister W: Proteasomes and other self-compartmentalizing proteases in prokaryotes. Trends Microbiol 1999, 7:88-92.

32. Rawlings ND, Morton FR, Barrett AJ: MEROPS: the peptidase database. Nucleic Acids Res 2006, 34:D270-D272.

33. Bochtler M, Ditzel L, Groll M, Huber R: Crystal structure of heat shock locus V (HsIV) from Escherichiacoli. Proc Natl Acad Sci U S A 1997, 94:6070-6074

34. Bochtler M, Hartmann C, Song HK, Bourenkov GP, Bartunik HD, Huber R: The structures of HsIU and the ATP-dependent protease HsIU-HsIV. Nature 2000, 403:800-805.

35. Ben-Zvi AP, Goloubinoff P: Review: mechanisms of disaggregation and refolding of stable protein aggregates by molecular chaperones. J Struct Biol 200I, I 35:84-93.

36. Glover JR, Lindquist S: Hsp I 04, Hsp70, and Hsp40: a novel chaperone system that rescues previously aggregated proteins. Cell 1998, 94:73-82.

37. Mogk A, Tomoyasu T, Goloubinoff P, Rudiger S, Roder D, Langen H, Bukau B: Identification of thermolabile Escherichiacoli proteins: prevention and reversion of aggregation by DnaK and CIpB. EMBO J 1999, I 8:6934-6949.

38. Motohashi K, Watanabe $Y$, Yohda M, Yoshida M: Heat-inactivated proteins are rescued by the DnaKJ-GrpE set and ClpB chaperones. Proc Natl Acad Sci U S A 1999, 96:7184-7I89.

39. Zolkiewski M: ClpB cooperates with DnaK, DnaJ, and GrpE in suppressing protein aggregation. A novel multi-chaperone system from Escherichiacoli. J Biol Chem 1999, 274:28083-28086.

40. Parsell DA, Kowal AS, Singer MA, Lindquist S: Protein disaggregation mediated by heat-shock protein Hsp 104. Nature 1994, 372:475-478.

4I. Schlee S, Groemping Y, Herde P, Seidel R, Reinstein J: The chaperone function of ClpB from Thermusthermophilus depends on allosteric interactions of its two ATP-binding sites. J Mol Biol 200I, 306:889-899.

42. Chuang SE, Burland V, Plunkett G III, Daniels DL, Blattner FR: Sequence analysis of four new heat-shock genes constituting the hsITS/ibpAB and hsIVU operons in Escherichiacoli. Gene 1993, 134:1-6.

43. Neuwald AF, Aravind L, Spouge JL, Koonin EV: AAA+: a class of chaperone-like ATPases associated with the assembly, operation, and disassembly of protein complexes. Genome Res 1999, 9:27-43.

44. Hung LW, Wang IX, Nikaido K, Liu PQ, Ames GF, Kim SH: Crystal structure of the ATP-binding subunit of an ABC transporter. Nature 1998, 396:703-707.

45. David JD, Wiesmeyer H: Control of xylose metabolism in Escherichiacoli. Biochim Biophys Acta 1970, 20 I:497-499.

46. Shamanna DK, Sanderson KE: Uptake and catabolism of Dxylose in Salmonellatyphimurium LT2. J Bacteriol 1979, 139:64-70.

47. Angen, Olsen JE, Bisgaard M: Further studies of the relationships among strains classified as taxon 15, taxon 18, taxon 20, (Pasteurella) granulomatis or the (Pasteurella) haemolyticacomplex in ruminants using quantitative evaluation of phenotypic data. Zentralbl Bakteriol 1997, 286:317-332.

48. Wissenbach U, Keck B, Unden G: Physical map location of the new artPIQMJ genes of Escherichiacoli, encoding a periplasmic arginine transport system. J Bacteriol 1993, I 75:3687-3688.

49. Song S, Park C: Organization and regulation of the D-xylose operons in Escherichiacoli K-I2: XyIR acts as a transcriptional activator. J Bacteriol 1997, I 79:7025-7032.

50. Lawrence JG, Ochman H: Amelioration of bacterial genomes: rates of change and exchange. J Mol Evol 1997, 44:383-397.
51. Marciel AM, Highlander SK: Use of operon fusions in Mannheimiahaemolytica to identify environmental and cis-acting regulators of leukotoxin transcription. Infect Immun 200I, 69:623I-6239.

52. Angen, Caugant DA, Olsen JE, Bisgaard M: Genotypic relationships among strains classified under the (Pasteurella) haemolytica-complex as indicated by ribotyping and multilocus enzyme electrophoresis. Zentralbl Bakteriol 1997, 286:333-354.

53. Angen, Aalbaek B, Falsen E, Olsen JE, Bisgaard M: Relationships among strains classified with the ruminant Pasteurellahaemolytica-complex using quantitative evaluation of phenotypic data. Zentralbl Bakteriol 1997, 285:459-479.

54. Altschul SF, Madden TL, Schaffer AA, Zhang J, Zhang Z, Miller W, Lipman DJ: Gapped BLAST and PSI-BLAST: a new generation of protein database search programs. Nucleic Acids Res 1997, 25:3389-3402.

55. Boeckmann B, Bairoch A, Apweiler R, Blatter MC, Estreicher A Gasteiger E, Martin MJ, Michoud K, O'Donovan C, Phan I, Pilbout S, Schneider M: The SWISS-PROT protein knowledgebase and its supplement TrEMBL in 2003. Nucleic Acids Res 2003, 3 I:365-370.

56. Tatusov RL, Fedorova ND, Jackson JD, Jacobs AR, Kiryutin B, Koonin EV, Krylov DM, Mazumder R, Mekhedov SL, Nikolskaya AN, Rao BS, Smirnov S, Sverdlov AV, Vasudevan S, Wolf YI, Yin JJ, Natale DA: The COG database: an updated version includes eukaryotes. BMC Bioinformatics 2003, 4:4I.

57. C. M, Jensen LJ, Snel B, Hooper SD, Krupp M, Foglierini M, Jouffre N, Huynen MA, Bork P: STRING: known and predicted proteinprotein associations, integrated and transferred across organisms. Nucleic Acids Res 2005, 33:D433-D437.
Publish with Biomed Central and every scientist can read your work free of charge

"BioMed Central will be the most significant development for disseminating the results of biomedical research in our lifetime. "

Sir Paul Nurse, Cancer Research UK

Your research papers will be:

- available free of charge to the entire biomedical community

- peer reviewed and published immediately upon acceptance

- cited in PubMed and archived on PubMed Central

- yours - you keep the copyright
BioMedcentral 